\title{
Electronic AVTA: Signal processing for automatic vocal transaction analysis
}

\author{
JAMES M. DABBS, JR. and MARK S. EVANS \\ Georgia State University, Atlanta, Georgia 30303
}

\begin{abstract}
An integrated circuit device that simplifies computer acquisition of the sound-silence patterns of vocalization in dyadic conversations is described. The unit converts analog voice signals into digital form, handles the problem of cross talk that occurs when persons seated close together are recorded on separate microphones, and adds a smoothing constant to fill in brief pauses within and between vocalizations.
\end{abstract}

There is a tradition of research on dyadic conversations that examines patterns of sound and silence. Each speaker's voice is scored as on or off at each moment in time, and the sequence and duration of the on-off signals are analyzed. Early research in this area (Chapple, 1949) was made more popular by Jaffe and Feldstein (1970), and summaries of recent findings are provided by Feldstein and Welkowitz (1978) and Seigman and Feldstein (1979). The equipment originally used for this research was cumbersome, but minicomputers and microcomputers now make it cheaper and easier to collect data (see Cappella \& Streibel, 1979; Dabbs, Evans, Hopper, \& Purvis, 1980).

A particular problem arises in trying to separate two voices inadvertently recorded through the same microphone. Even when two subjects seated near each other are given separate lapel or lavalier microphones and separate recording channels, there is some "cross talk," or spilling over of one subject's voice into the microphone and recording channel of the other subject. A computer responding to the presence or absence of a voice on an audio channel cannot tell whether the voice comes from a subject who belongs on the channel or from another (overheard) subject who primarily belongs on another channel. Cassotta, Feldstein, and Jaffe (1964) and Jaffe and Feldstein (1970) described an electronic device to cancel the cross talk between microphones. In this paper, we describe an integrated circuit device to accomplish the same purpose.

The device takes input from the two voice channels of a stereo recorder. It amplifies the input, cancels cross talk, adds a smoothing constant to the end of each utterance, converts the flow of sound and silence into a series of binary on-off signals, and actuates light-emitting diodes so the operator can monitor the output. The unit operates on a \pm 5 - $\mathrm{V}$ power supply, although it would probably also work with a higher voltage power supply.

A diagram of the device is shown in Figure 1. The

Mark Evans is now at Bell Telephone Laboratories, Holmdel, New Jersey. The authors wish to thank William Nelson for help in building the device described here. functions of integrated circuits IC1 through IC8 and variable resistors $\mathrm{R} 1$ through $\mathrm{R} 8$ are described here. IC1 and IC2 provide initial amplification. R1 and R2 can be used to adjust the amplification to make the two incoming signal levels approximately the same; this may be necessary if the recording is louder on one channel than on the other. An external stereo amplifier can be used to supplement or replace IC1 and IC2. If an oscilliscope is available, it can be used to monitor the output of IC1 and IC2. In using the device, we have usually adjusted the amplification so that the outputs of IC1 and IC2 are in the 2- to 4-V range.

IC3 and IC4, along with R3 and R4, cancel the cross talk between microphones. A portion of the audio signal from each channel goes into the inverting input of the IC amplifier in the other channel, in effect subtracting any appearance of the voice from the channel in which it was not intended to appear. Although R3 and R4 can be set to subtract varying amounts of a signal from its nonintended channel, in practice we have usually set these resistors to zero and subtracted the full value of the signal. Adjusted in this fashion, the unit slightly attenuates the voice intended to appear on that channel, when both subjects speak at once, but it still indicates that both subjects are speaking at once.

IC5 and IC6 convert the audio signal to digital form. The outputs of IC5 and IC6 remain at ground level until their input signals rise above the trigger voltage level set at the inverting inputs of IC5 and IC6, at which point the outputs rise to $+5 \mathrm{~V}$. The trigger level is set using the voltage dividers R7 and R8; a convenient level is $.05 \mathrm{~V}$.

IC7 allows the user to introduce a delay in the offset of a voice signal and thereby smooth over short periods of silence. The length of this delay is determined by R7 and R8, and it is usually set to $300 \mathrm{msec}$. The delay is calibrated by introducing a signal into the device, then terminating the input signal and measuring the delay between termination of the input and termination of the output, and adjusting R7 and R8 until the delay on each channel is equal to $300 \mathrm{msec}$. IC8 continuously retriggers IC7 to keep the output signal on during long 


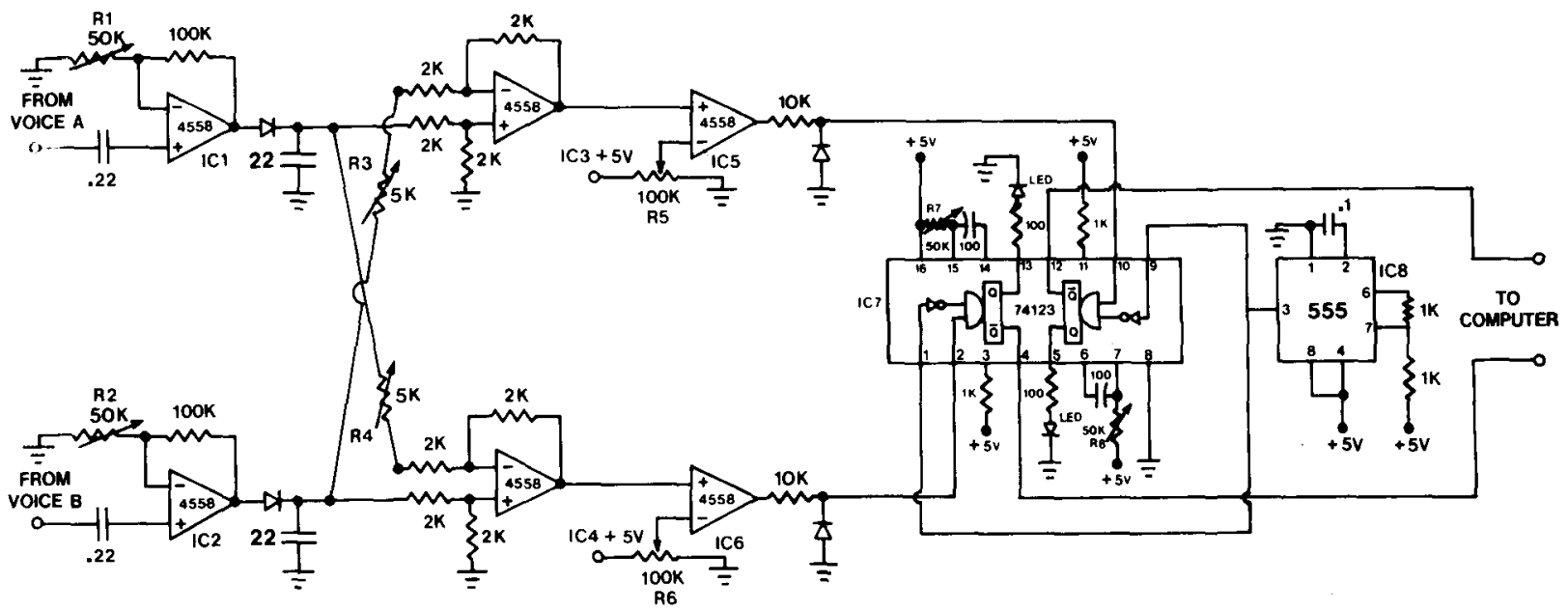

Figure 1. Diagram of signal processing device. Operational amplifiers other than XR4558s could be used. The $4558 \mathrm{~s}$ are packaged in pairs, and if space is at a premium, the whole device can be built on a $2 \times 6$ in. $(5 \times 15 \mathrm{~cm})$ protostrip. All capacitance values are in microfarads.

vocalizations. The $300-\mathrm{msec}$ "fill-time" constant is the same as that used by Dabbs et al. (1980), although Jaffe and Feldstein (1970) used a constant of about $200 \mathrm{msec}$, and Feldstein (Note 1) currently uses a 50 -msec constant. Other investigators may wish to adjust R7 and R8 to produce fill times of less than 300 msec.

The output of the device is normally $5 \mathrm{~V}$ and goes to zero when a subject speaks. If the computer receiving the input requires a positive rather than a negative signal, this can be arranged by changing connections at the $Q$ and not-Q outputs of IC7, or, alternatively, a switch can be arranged at the output. The light-emitting diodes allow the researcher to monitor the operation of the device while listening to the voice inputs, to make sure the output "looks like" the conversation sounds. The light signals are helpful in adjusting initial amplification levels so that the device works properly.

\section{REFERENCE NOTE}

1. Feldstein, S. Personal communication, August 13, 1982.

\section{REFERENCES}

Cappella, J. N., \& Streibel, M. J. Computer analysis of talk-silence sequences: The FIASSCO system. Behavior Research Methods \& Instrumentation, 1979, 11, 384-392.

Cassotta, S., Feldstein, S., \& Jaffe, J. AVTA: A device for automatic vocal transaction analysis. Journal of the Experimental Analysis of Behavior, 1964, 7, 99-104.

Chapple, E. D. The interaction chronograph: Its evolution and present application. Personnel, 1949, 25, 295 -307.

Dabbs, J. M., Jr., Evans, M. S., Hopper, C. H., \& Purvis, J. A. Self-monitors in conversation: What do they monitor? Journal of Personality and Social Psychology, 1980, 39, 278284.

Feld8tein, S., \& Welkowitz, J. A chronography of conversation: In defense of an objective approach. In A. W. Siegman \& S. Feldstein (Eds.), Nonverbal behavior and communication. Hillsdale, N.J: Erlbaum, 1978.

Jaffe, J., \& Feldotein, S. Rhythms of dialogue. New York: Academic Press, 1970.

Siegman, A. W., \& Feldstein, S. (Eds.). Of speech and time: Temporal speech patterns in interpersonal contexts. Hillsdale, N.J: Erlbaum, 1979.

(Received for publication April 30, 1982; revision accepted August 24, 1982.) 